\title{
DYNAMIC ADAPTATION IN CONVECTION - DIFFUSION EQUATIONS
}

\author{
A. V. MAZHUKIN ${ }^{1}$
}

\begin{abstract}
With the example of solving some known modeling problems the features of constructing grids adapted to the solution of parabolic equations are considered. Convection-diffusion problems are described by nonlinear Burgers and Buckley Leverette equations.

A detailed analysis of the differential approximations and numerical results shows that the idea of using an arbitrary time-dependent system of coordinates for adapted grid generation in combination with the principle of quasi-stationarity makes the dynamic adaptation method universal, effective, and algorithmically simple.

Universality is achieved due to the use of an arbitrary time-dependent system of coordinates that moves at a velocity determined by the unknown solution. This universal approach makes it possible to generate adapted grids for time-dependent problems of mathematical physics with various mathematical features. Among these features are large gradients, and propagation of weak and strong discontinuities in known nonlinear transfer problems.

The efficiency is determined by automatically fitting the velocity of the moving nodes to the dynamics of the solution. The close relationship between the adaptation mechanism and the structure of parabolic equations allows one to automatically control nodes motion of nodes so that their trajectories do not intersect.

The simplicity of the algorithm is achieved due to the general approach to the adaptive grid generation that is independent of the form and type of the differential equations.
\end{abstract}

2000 Mathematics Subject Classification: 37K10, 35Q53, 00A79.

Keywords: dynamic adaptation, principle of quasi-stationarity, grids adapted to the solution, parabolic equation, differential approximation, finite difference scheme, nonlinear convection - diffusion equation.

\section{Introduction}

Accuracy of a numerical solution to a partial differential equation depends on how well the distribution of the grid points corresponds to the features of the unknown solution. Optimal grids can be generated using various adaptation methods (see [1-5]). The application of one or another adaptation method depends on several factors of which the main are the type of the equation or the system of equations, their dimensionality, the degree of nonlinearity, the presence of moving boundaries, and others.

The problem of optimal distribution of the grid points is especially pressing in nonlinear time-dependent problems of mathematical physics whose solutions contain a strongly non-uniformly scaled spatial varibles. Such features of the solution as large gradients and

\footnotetext{
${ }^{1}$ Institute of Mathematical Modeling, Russian Academy of Sciences, Miusskaya sq. 4a, 125047 Moscow, Russia. E-mail: immras@orc.ru
} 
transient layers or discontinuity fronts can appear near one of the boundaries or in the interior of the domain and then spread over the entire computational domain. In such situations, it is impossible to generate a grid with the optimal distribution of nodes in advance. For that reason, grids adaptable to the unknown solution have found wide use. In the dynamic adaptation methods $[6,7]$ controlled distribution of the grid points is achieved by using information about the dynamics of the unknown solution. This allows one to concentrate more grid points in the regions where the solution undergoes sharp changes. A close relationship between the dynamics of the solution and the location of the grid points leads to the relocation of these points at each time layer. This places more stringent requirements upon the matching of the relocation of grid points to the dynamics of the numerical solution and upon the degree of automation of the grid generation. Therefore, the algorithms that do not use fitting parameters (see [8]) have an advantage.

This paper is devoted to the definition of the general mechanism of generation of grids that can dynamically adapt themselves to the solutions of parabolic convection-diffusion equations (Buckley — Leverette and Burgers equations). In these problems, the distribution of the grid points depends on both the moving boundaries and the specific features of the solution in the interior of the domain.

Convection-diffusion problems appear in many mathematical models that form the basis for fluid mechanics. These models describe two main mechanisms of mass and energy transfer-diffusion and convection. Depending on external conditions, each of these mechanisms can be dominating. To assess the degree of dominance of a particular process, dimensionless parameters (the so-called Peclet $(\mathrm{Pe})$ or Reynolds (Re) numbers) are used. When $\mathrm{Pe} \ll 1(\mathrm{Re} \ll 1)$, the diffusive process is dominating; when $\mathrm{Pe} \gg 1(\mathrm{Re} \gg 1)$, the convective process is dominating. When convecting transfer is strongly dominating, the class of singularly perturbed nonlinear mathematical models with a small parameter $\mathrm{Pe}^{-1}$ or $\mathrm{Re}^{-1}$ at higher order derivative are obtained. In the time-dependent singularly perturbed models, sharp solution change domains that propagate in the form of various fronts and transient layers can appear.

From the computational point of view, the singularly perturbed problems are difficult. In particular, the finite difference schemes used to approximate the convection-diffusion equations usually have a wide variation, which is suppressed by using special techniques.

\section{Statement of the problem}

Both equations have the same structure and only differ by the form of the nonlinear operator $P(u)$ :

$$
\begin{gathered}
\frac{\partial u}{\partial t}+\frac{\partial P(u)}{\partial x}=\frac{\partial}{\partial x} \mu \frac{\partial u}{\partial x}, \\
x=x_{0}: \quad g_{0}\left(t, x_{0}, u(x), \frac{\partial u(x)}{\partial x}, \ldots\right), \\
x=x_{r}: \quad g_{r}\left(t, x_{r}, u(x), \frac{\partial u(x)}{\partial x}, \ldots\right), \\
t=0: \quad u(x, 0)=u^{0}(x) .
\end{gathered}
$$

Here, $P(u)=u^{2} /\left[u^{2}+\alpha(1-u)^{2}\right]$ - for the Buckley — Leverette equation, $P(u)=u^{2} / 2-$ for the Burgers equation, and $\mu \sim(\mathrm{Re})^{-1}$ or $\mu \sim(\mathrm{Pe})^{-1}$ — (the small parameter multiplying 
the higher order derivative) is interpreted as diffusion in the Buckley - Leverette equation and as viscosity in the Burgers equation. Due to the small multiplier of the higher order derivative, both equations belong to the class of singularly perturbed equations. The solution of such equations is complicated because regions of sharp variation of the solution may appear under some boundary conditions. The difference schemes used to solve convection-diffusion equations in the case where convection prevails usually have a wide variation, which results in the appearance of parasitic oscillations when the solution changes in a jump. We demonstrate the efficiency of the dynamic adaptation method for solving equations of this class.

\section{Arbitrary time-dependent system of coordinates}

The dynamic adaptation method is based on the procedure of passing to an arbitrary timedependent system of coordinates. Such a system makes it possible to formulate the problem of generation and adaptation of computational grids at the differential level. More precisely, some of the differential equations in the resulting mathematical model describe the physical processes and the others describe the the behavior of the grid points [8]. The change to the arbitrary time-dependent system of coordinates is performed by an automatic transformation of coordinates using the unknown solution.

2.1. Change of variables. Following [9-12], we replace the physical space $\Omega_{x, t}$ with the Euler coordinates $(x, t)$ by a computational space with an arbitrary time-dependent system of coordinates $\Omega_{q, \tau}$ in the variables $(q, \tau)$. This replacement can be performed using the change of variables of the general form $x=f(q, \tau), t=\tau$ that has a one-valued nonsingular inverse $q=\varphi(x, t), \tau=t$. The partial derivatives of the dependent variables when we change the coordinates are related by

$$
\frac{\partial .}{\partial t}=\frac{\partial .}{\partial \tau}+\frac{Q}{\psi} \frac{\partial .}{\partial q}, \quad \frac{\partial \cdot}{\partial x}=\frac{1}{\psi} \frac{\partial .}{\partial q}, \quad \frac{\partial .^{2}}{\partial x^{2}}=\frac{1}{\psi} \frac{\partial}{\partial q} \frac{1}{\psi} \frac{\partial}{\partial q}
$$

where $\psi=\partial x / \partial q$ is the Jacobian of the inverse transformation, $Q$ is the transformation function describing the velocity of the time-dependent system of coordinates. It is unknown a priori and must be found.

Using the change of variables of the general form and relations (2), we represent the differential model $(1)$ in the variables $(q, \tau)$ in the form

$$
\begin{gathered}
\frac{\partial u}{\partial \tau}+\frac{Q}{\psi} \frac{\partial u}{\partial q}+\frac{1}{\psi} \frac{\partial P(u)}{\partial q}=\frac{1}{\psi} \frac{\partial}{\partial x} \mu \frac{\partial u}{\partial x}, \\
\frac{\partial x}{\partial \tau}=-Q \\
q_{0}<q<q_{r}, \quad \tau>0,
\end{gathered}
$$

where (4) - is the inverse transformation equation involving the transformation function $Q$. The function $Q$, which is unknown a priori and must be found, describes the velocity of the time-dependent system of coordinates. The inverse transformation equation written in the form

$$
\frac{\partial \psi}{\partial \tau}=-\frac{\partial Q}{\partial q}
$$


is more convenient. It can be obtained by differentiating Eq. (4) with respect to $q$. Simple manipulations reduce system (3), (4) to the conservative form

$$
\begin{gathered}
\frac{\partial(\psi u)}{\partial \tau}+\frac{\partial(u Q)}{\partial q}+\frac{\partial P(u)}{\partial q}=\frac{\partial}{\partial x} \mu \frac{\partial u}{\partial x} \\
\frac{\partial \psi}{\partial \tau}=-\frac{\partial Q}{\partial q}
\end{gathered}
$$

Thus, as a result of passing to the arbitrary time-dependent system of coordinates, the original differential model is transformed to an extended system of differential equations that includes an additional equation (4) or (6). Its type, properties, and the form of the boundary conditions depend on $Q$. At this stage of the reasoning, the function $Q$ is unknown. As soon as it is determined, Eq. (6) is used to generate a grid adapted to the solution. Its difference analog describes the dynamics of the grid points, and $Q$ controls the relocation of the grid points in coordination with the dynamics of the unknown solution. The coordination is achieved by introducing the dependence of $Q$ on the unknown solution. However, since this solution is not known a priori, the problem of determining the optimal transformation function $Q$ that guarantees a complete coordination of the adaptation mechanism with the solution arises. If the coordination is incomplete, some fitting coefficients are included in the control function that help to reduce the degree of incoordination. However, the use of fitting coefficients in the adaptation method indicates that it is imperfect.

2.2. The principle of quasi-stationarity. The function $Q$ determines a concrete change of coordinates and the method of controlling the relocation of the grid points. It has already been mentioned that finding the optimal function Qthat ensures the relocation of the grid points fitted to the solution is a very important point in the dynamic adaptation method. The perfect fitting implies several requirements of which the most important are the following ones:

- distribution of the grid points depending on the specific features of the unknown solution;

- availability of a reliable automatic control of the grid points relocation so as to prevent the intersection of their trajectories in the course of adaptation;

- complete automation of the adaptation process, which assumes the absence of fitting parameters that change from variant to variant;

- possibility of changing the number of grid points in the course of the computations, which assumes that new nodes may be generated if needed and redundant nodes can be eliminated.

Practical applications of dynamic adaptation (see [18-20]) show that, in order to satisfy the above requirements and ensure perfect fitting, the structure of $Q$ must be closely related to the structure of the original equation (see [11]) or the system of equations (see $[19,20]$ ) that describe the physical process. Such a relation can be obtained using the principle of quasi-stationarity (see [5]). The idea of this principle is as follows.

When changing the coordinates, it is implicitly assumed that the time-dependent system of coordinates is chosen such that the time derivatives of the solution in the new coordinates are much smaller than in the original coordinates. A logical completion of this assumption is 
the requirement that the time derivatives vanish or, at least, are very small. The smallness of the time derivatives is equivalent to the requirement of the quasi-stationarity of the processes in the new system of coordinates. This point is very important because it is well known that the time derivatives play an important role in the dissipative and variation properties of finite difference schemes.

Assume that we have found a system of coordinates in which $\partial u / \partial \tau=0$. Then, the equation takes the form

$$
\frac{Q}{\psi} \frac{\partial u}{\partial q}+\frac{1}{\psi} \frac{\partial P(u)}{\partial q}=\frac{\mu}{\psi} \frac{\partial}{\partial q} \frac{1}{\psi} \frac{\partial u}{\partial q}
$$

in terms of these coordinates. Hence, we find the function $Q$ in the form

$$
Q=-\left(\frac{\partial P(u)}{\partial u}-\mu \frac{\partial}{\partial q}\left(\frac{1}{\psi}\right)-\frac{\mu}{\psi} \frac{\partial^{2} u / \partial q^{2}}{(\partial u / \partial q+\text { re } * \operatorname{sign}(\partial u / \partial q))}\right)
$$

where re $\ll 1$ - is a regularization constant that places a lower bound on the value of the derivative $\partial u / \partial q$ when it is small.

Let us analyze (7). After differentiation, the first term is either the solution $u$ or its derivative $\partial u / \partial q$ or their combination. Upon a difference approximation, this term produces a strong contractive effect on the grid points. The third term has a similar effect. However, as this term is a ratio of the derivatives, its effect is much weaker compared to the effect of the first term; for that reason, it is not always taken into account. The second term produces a repulsive effect on the grid points; it provides a mechanism for automatic control of the grid points relocation so as to prevent the intersection of their trajectories. When the distance between two adjacent grid points $(i, i+1)$ tends to zero, the function $\psi_{i+1 / 2}$, for the corresponding grid cell also tends to zero. The term $\frac{\partial}{\partial q}\left(\frac{1}{\psi}\right)$ grows sharply $\left(\right.$ as $\left.1 / \psi^{2}\right)$, and the repulsive effect of the function $Q$ increases, thus preventing the Jacobian of the transformation function from vanishing and the grid cells from collapsing.

2.3. Initial and boundary conditions. As a result of passing to an arbitrary timedependent system of coordinates, the coordinates $x_{i}^{j}$ of the grid points become unknown in addition to the grid functions $u_{i}^{j}$. To find them, the extended mathematical model (5)-(7) includes the partial differential equation (6).

Transformation of the original equation (2) into the extended system of differential equations (5)-(7) requires the corresponding extension of the boundary and initial conditions; that is, the quantities $\psi(0, q), Q\left(\tau, q_{0}, Q(\tau, q(r))\right)$ should be additionally specified.

The initial conditions at $\tau=\tau_{0}=0$ are determined using the following considerations. The function $\psi=\partial x / \partial q$, which is the Jacobian of the transformation, is the factor by which the geometric size of the original domain or of an individual grid size h change compared to the original values at $t=0$. Assuming that passing to the computational domain is not associated with the deformation of the original domain, we conclude that the following conditions must be satisfied at the initial instant of time

$$
\tau=\tau_{0}: \quad u\left(\tau_{0}, q\right)=u_{0} \quad \psi\left(\tau_{0}, q\right)=1 .
$$

The boundary conditions for Eq. (5) take the form

$$
q=q_{0}: \quad g_{0}\left[\tau, q, u(q), \frac{1}{\psi} \frac{\partial u}{\partial q}\right]=0
$$




$$
q=q_{r}: \quad g_{0}\left[\tau, q, u(q), \frac{1}{\psi} \frac{\partial u}{\partial q}\right]=0 .
$$

When formulating the boundary conditions for the inverse transformation equation (6), the conditions that are common for all types of $Q$ must be taken into account. These conditions are as follows. All the fixed boundaries are associated with the condition $Q(\tau, q)=$ 0 . Any other condition $Q(\tau, q) \neq 0$ corresponds to a moving boundary of the domain. Using a numerical solution of some model problems describing the processes of nonlinear heat conduction and convection - diffusion as examples, we show that the principle of quasi-stationarity makes it possible to determine the transformation functions $Q$ that ensure complete agreement of the adaptation mechanism with the unknown solution in parabolic equations.

\section{Nonlinear convection - diffusion equations}

The use in relation (1) of the expression $P(u)=u^{2} /\left[u^{2}+\alpha(1-u)^{2}\right]$ leads to the parabolic Buckley - Leverette equation [22], which is the basic equation in the model problem describing the motion of a two-phase immiscible fluid (for example, oil-water) in a porous medium; this model describes the convective transport and diffusion.

Using relation $P(u)=u^{2} / 2$ turns Eq. (1) into a complete Burgers equation, which provides a model for the boundary layer equations, the Navier - Stokes equations brought to the parabolic form, and the complete Navier — Stokes equations (see [21]).

3.1. Buckley - Leverette equation. The complete statement of Buckley — Leverette problem has form

$$
\begin{gathered}
\frac{\partial u}{\partial t}+\frac{\partial\left(\frac{u^{2}}{u^{2}+\alpha(1-u)^{2}}\right)}{\partial x}=\frac{\partial}{\partial x} \mu \frac{\partial u}{\partial x}, \\
x_{0}<x<\infty, \quad t>0, \\
t=0: \quad u(x, 0)=u^{0}(x), \\
x=x_{0}: \quad g_{0}\left(t, x_{0}, u(x) \frac{\partial u(x)}{\partial x}, \ldots\right)=0, \\
x=x_{r}: \quad g_{r}\left(t, x_{r}, u(x) \frac{\partial u(x)}{\partial x}, \ldots\right)=0 .
\end{gathered}
$$

Using the change of variables of the general form and applying relation (2) and defining

$$
W(u)=P(u)-\frac{\mu}{\psi} \frac{\partial u}{\partial q},
$$

we rewrite Eq. (10) in terms of variables in the conservative form

$$
\begin{gathered}
\frac{\partial(\psi \cdot u)}{\partial t}=-\frac{\partial(Q \cdot u)}{\partial q}-\frac{\partial W}{\partial q}, \\
\frac{\partial \psi}{\partial \tau}=-\frac{\partial Q}{\partial q}, \quad \psi=\frac{\partial x}{\partial q}, \\
q_{0}<q<q_{r}, \quad \tau>0,
\end{gathered}
$$




$$
\tau=0:\left\{\begin{array}{l}
u(0, q)=u^{0}(q) \\
\psi(0, q)=\psi^{0}(q),
\end{array} \quad q=0:\left\{\begin{array}{l}
u(\tau, 0)=g_{0}(t) \\
Q(\tau, 0)
\end{array} \quad q=\infty:\left\{\begin{array}{l}
u(\tau, \infty) \\
Q(\tau, \infty) .
\end{array}\right.\right.\right.
$$

The function $Q$ is defined by the quasi-stationarity condition

$$
Q(u)=-\left(\frac{2 \alpha u(1-u)}{\left[u^{2}+\alpha(1-u)^{2}\right]^{2}}-\frac{\partial}{\partial q}\left(\frac{\mu}{\psi} \frac{\partial u}{\partial q}\right)\right)
$$

or

$$
Q=-\left(\frac{\partial P(u)}{\partial u}-\frac{\partial}{\partial q}\left(\frac{\mu}{\psi}\right)-\frac{\mu}{\psi} \frac{\partial^{2} u}{\partial q^{2}} /\left(\frac{\partial u}{\partial q}+\mathrm{re}\right)\right)
$$

where

$$
\frac{\partial P(u)}{\partial u}=\frac{2 \alpha u(1-u)}{\left[u^{2}+\alpha(1-u)^{2}\right]^{2}}
$$

3.2. Analysis of the differential approximations. In the discrete space $\omega_{h}^{\Delta \tau}$ consider the family of five-point schemes for the time-independent equation (13). The analytical investigation of the quality of these schemes is much easier than the investigation of the schemes for the time-dependent equation (11).

The scheme with the central difference quotients has the form

$$
Q_{i} \frac{u_{i+1}-u_{i-1}}{2 h}+\frac{P_{i+1}-P_{i-1}}{2 h}-\frac{1}{h}\left(\frac{\mu}{\Psi_{i+1 / 2}} \frac{u_{i+1}-u_{i}}{h}-\frac{\mu}{\Psi_{i-1 / 2}} \frac{u_{i}-u_{i-1}}{h}\right)=0,
$$

the scheme based on the forward difference quotients is

$$
Q_{i} \frac{u_{i+1}-u_{i}}{h}+\frac{P_{i+1}-P_{i}}{2}-\frac{1}{h}\left(\frac{\mu}{\Psi_{i+1 / 2}} \frac{u_{i+1}-u_{i}}{h}-\frac{\mu}{\Psi_{i-1 / 2}} \frac{u_{i}-u_{i-1}}{h}\right)=0,
$$

and the scheme based on the backward difference quotients is

$$
Q_{i} \frac{u_{i}-u_{i-1}}{h}+\frac{P_{i}-P_{i-1}}{2}-\frac{1}{h}\left(\frac{\mu}{\Psi_{i+1 / 2}} \frac{u_{i+1}-u_{i}}{h}-\frac{\mu}{\Psi_{i-1 / 2}} \frac{u_{i}-u_{i-1}}{h}\right)=0 .
$$

For schemes (15)-(17) we write their differential approximations, which only differ by the coefficients on the right-hand sides

$$
Q \frac{\partial u}{\partial q}-\frac{\partial}{\partial q}\left(\frac{k(u)}{\psi} \frac{\partial u}{\partial q}\right)=\delta_{n} \frac{\partial u}{\partial q}+\alpha_{n} \frac{\partial^{2} u}{\partial q^{2}}+\beta_{n} \frac{\partial^{3} u}{\partial q^{3}}+\gamma_{n} \frac{\partial^{4} u}{\partial q^{4}}, \quad n=1,2,3 .
$$

For scheme (15) on the central difference quotients, $n=1$, we have:

$$
\begin{gathered}
\delta_{1}=-\frac{h^{2}}{24}\left(\left(4 \frac{\partial^{3} P}{\partial u^{3}}\right)\left(\frac{\partial u}{\partial q}\right)^{2}-\frac{\partial^{3}}{\partial q^{3}}\left(\frac{\mu}{\psi}\right)\right), \quad \alpha_{1}=-\frac{h^{2}}{8}\left(4 \frac{\partial^{2} P}{\partial u^{2}} \frac{\partial u}{\partial q}-\frac{\partial^{2}}{\partial q^{2}}\left(\frac{\mu}{\psi}\right)\right), \\
\beta_{1}=-\frac{h^{2}}{6}\left(Q+\frac{\partial P}{\partial u}-\frac{\partial}{\partial q}\left(\frac{\mu}{\psi}\right)\right), \quad \gamma_{1}=\frac{h^{2}}{12} \frac{\mu}{\psi} .
\end{gathered}
$$

For the schemes with the forward difference quotients (16), $n=2$ and backward quotients (17), $n=3$ the differential approximations only differ by the sign on the right-hand side

$$
Q \frac{\partial u}{\partial q}+\frac{\partial P(u)}{\partial q}-\frac{\partial}{\partial q} \frac{\mu}{\psi} \frac{\partial u}{\partial q}=\delta_{2,3} \frac{\partial u}{\partial q}+\alpha_{2,3} \frac{\partial^{2} u}{\partial q^{2}}+\beta_{2,3} \frac{\partial^{3} u}{\partial q^{3}}+\gamma_{2,3} \frac{\partial^{4} u}{\partial q^{4}} .
$$


Here

$$
\begin{gathered}
\delta_{2,3}=\delta_{1}+\delta_{0}, \quad \alpha_{2,3}=\alpha_{0}+\alpha 1, \quad \beta_{2,3}=\beta_{1}, \quad \gamma_{2,3}=\gamma_{1}, \\
\delta_{0}= \pm \frac{h}{2} \frac{\partial^{2} P}{\partial u^{2}} \frac{\partial u}{\partial q}, \quad \alpha_{0}= \pm \frac{h}{2}\left(Q+\frac{\partial P}{\partial u}\right) .
\end{gathered}
$$

Here, the index 2 corresponds to the equation for scheme (16), and the index 3 corresponds to the differential approximation for scheme (17). The upper sign in $\delta_{2,3}$ and $\alpha_{2,3}$ corresponds to the index 2 , and the lower sign to the index 3 . Let us analyze the coefficients $\alpha$ and $\beta$ multiplying the second-order and the third-order derivatives; they characterize, respectively, the dissipation and the variation of the difference schemes. Both coefficients depend on the adaptation parameter, which indicates that the dissipation and variation of the schemes depend on the adaptation method, and a proper choice of the control function $Q$ can increase or decrease the dissipation and variation. The coefficient $\beta$ has the same form for all the three families of schemes; it vanishes if $Q$ is chosen as

$$
Q=-\left(\frac{\partial P}{\partial u}-\frac{\partial}{\partial q}\left(\frac{\mu}{\psi}\right)\right)
$$

Expression (20) is shorter by one term than (12), and it allows us to completely eliminate the internal variation of the schemes.

Scheme (15) based on the central difference quotients has the minimal variation among the tree schemes under examination. In the corresponding differential approximation (18), the coefficient of the dissipative term $\alpha_{1}$ is proportional to $h^{2}$. Taking into account the choice of $Q$ in the form (20), scheme (15) is formally the best one: it has a small scheme viscosity and no variation. In the second-order difference schemes with fixed grid points for convection diffusion equations, small dissipation plays a negative role because a small scheme viscosity cannot suppress parasitic oscillations of the solution since the dispersive component is not compensated. In schemes (16) and (17) of the first order $O(h)$, the dispersive component is proportional to $h$ and $\alpha_{2,3} \approx h$. However, the scheme viscosity is not large in this case and does not play as a significant role as in the schemes with fixed grid points. The coefficients $\alpha_{2}$ and $\alpha_{3}$ in Eq. (19) are a sum of $\alpha_{1} \sim h^{2}$ and an addition which depends on the transformation function $Q$. When $Q$ is given by (20), i. e., is proportional to the diffusion or viscosity coefficient $\mu$, we have

$$
\alpha_{0}=\mp \frac{h}{2} \frac{\partial}{\partial q}\left(\frac{\mu}{\psi}\right)
$$

In the most interesting cases where $\mu$ is very small, $\alpha_{0}$ is vanishingly small and the quality of schemes (16) and (17) is similar to scheme (15). For large $\mu$, the scheme viscosity is of little importance because a large diffusion or viscosity prevents the formation of steep gradients of the solution.

Thus, the analysis of the differential approximation shows that, as in the nonlinear heat conduction problems, the dynamic adaptation considerably improves the quality of the difference schemes. The grid refinement in the region where the gradients are large reduces the scheme viscosity and practically eliminates the variation of the difference schemes. The differential approximation can be also used to determine the optimal transformation function $Q$ because the results are close to those obtained using the quasi-stationarity principle.

3.3. Numerical solution of model problems. Consider two typical statements of convection - diffusion problems that use the Buckley - Leverette equation (see [22]) and the Burgers equation (see [23]). 
3.3.1. Buckley - Leverette equation. Define

$$
W(u)=P(u)-\frac{\mu}{\Psi} \frac{\partial u}{\partial q}
$$

and write the Buckley - Leverette equations in terms of the variables $(q, \tau)$ in the conservative form:

$$
\begin{gathered}
\frac{\partial(\psi u)}{\partial t}=-\frac{\partial(Q u)}{\partial q}-\frac{\partial W}{\partial q} \\
\frac{\partial \psi}{\partial t}=-\frac{\partial Q}{\partial q}, \quad q_{0}<q<q_{\infty}, \quad \tau>0
\end{gathered}
$$

where

$$
P(u)=\frac{u^{2}}{u^{2}+\alpha(1-u)^{2}}, \quad Q(u)=-\left(\frac{2 \alpha u(1-u)}{\left(u^{2}+\alpha(1-u)^{2}\right)^{2}}-\frac{\mu}{\psi} \frac{\partial u}{\partial q}\right)
$$

where $u$ characterizes the fluid saturation, $\mu$ has the sense of the diffusion coefficient, $\alpha$ is the parameter characterizing the mobility of one fluid relative to the other, and $u^{0}(q), \psi^{0}(q)$, $g_{0}$ and $g_{\infty}$ are known functions.

The representation of the Buckley - Leverette equation in form (21) makes it possible to use for the numerical solution the family of implicit conservative difference schemes:

$$
\begin{gathered}
(\psi u)_{i}^{j+1}=(\psi u)_{i}^{j}-\frac{\Delta \tau^{j}}{h}\left((1-\delta)\left(W_{i+1 / 2}-W_{i-1 / 2}+(u Q)_{i+1 / 2}-(u Q)_{i-1 / 2}\right)^{j}+\right. \\
\left.+\delta\left(W_{i+1 / 2}-W_{i-1 / 2}+(u Q)_{i+1 / 2}\right)^{j+1}\right) .
\end{gathered}
$$

In this case, the quantities $Q(u), W(u)$ are approximated by the finite difference relations

$$
\begin{aligned}
& W_{i+1 / 2}=\left(\frac{u^{2}}{\left(u^{2}+\alpha(1-u)^{2}\right)}\right)_{i+1 / 2}-\left(\frac{\mu}{\psi}\right)_{i+1 / 2} \frac{u_{i+1}-u_{i}}{h}, \\
& Q_{i}=-\left(\left(\frac{2 \alpha u(1-u)}{\left(u^{2}+\alpha(1-u)^{2}\right)^{2}}\right)_{i}-\frac{\mu}{h}\left(\frac{1}{\Psi_{i+1 / 2}}-\frac{1}{\Psi_{i-1 / 2}}\right)\right),
\end{aligned}
$$

In the calculations, we used the following values of the functions and parameters:

$$
\begin{gathered}
u^{0}(q)=e^{-10 q}, \quad \psi^{0}(q)=1, \quad g_{0}(\tau)=1, \\
g_{\infty}(\tau)=0, \quad \mu=10^{-5}, \quad \alpha=0.25,2.5
\end{gathered}
$$

3.3.2. Modeling results. First, for comparison, we solved Eq. (10) subject to conditions (27) on a grid with $N=500$ fixed nodes. The results obtained using schemes of the first order $O\left(\Delta \tau+h^{2}\right)$ and of the second order $O\left(\Delta \tau^{2}+h^{2}\right)$ are presented in Figs. 3.1 and 3.2. 
The schemes of the first order yield a solution that is significantly smoothed near the front (Fig. 3.1) and the schemes of the second order yield a poor solution because of the large variation (Fig. 3.2).

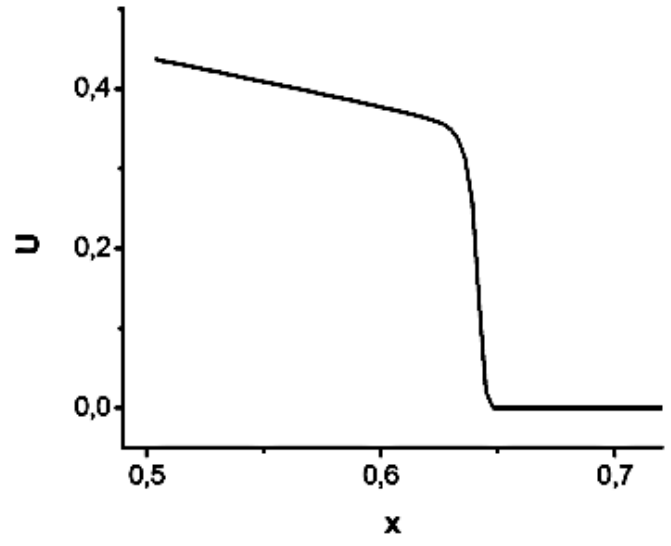

Fig. 3.1.

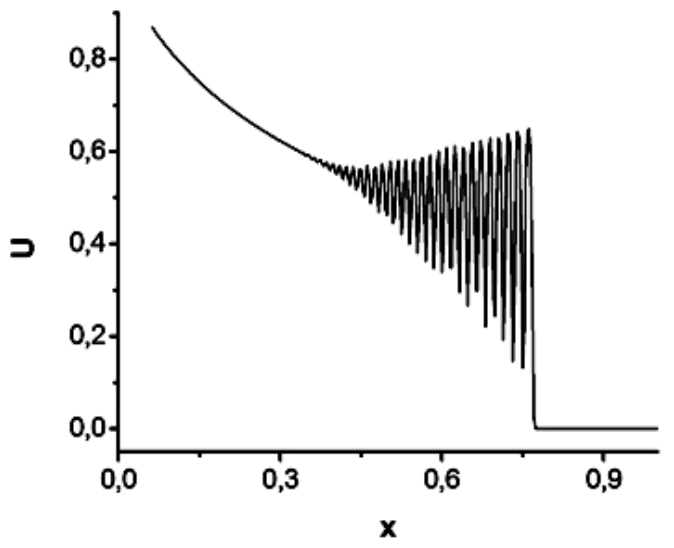

Fig. 3.2.

The application of dynamically adapted grids for solving problem (21)-(23), (27) using the difference schemes (24), (25), (26) of the first and second orders makes it possible to obtain solutions that are not smoothed and have no parasitic oscillations using only 1020 grid points. Figures 3.3, 3.4 show the spatial distribution of the function $u(x)$ and the distribution of the dimensionless grid size $\psi(x)$ for various instants of time for $\alpha=0.25$, $\psi=10^{-5}$, and $N=16$.

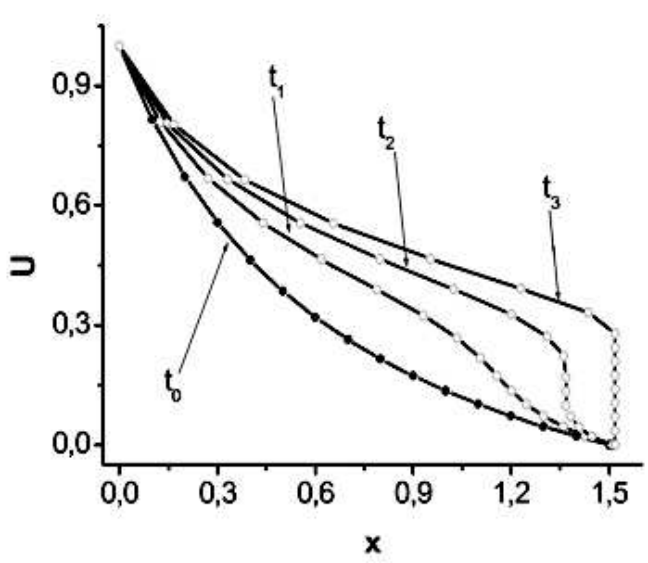

Fig. 3.3.

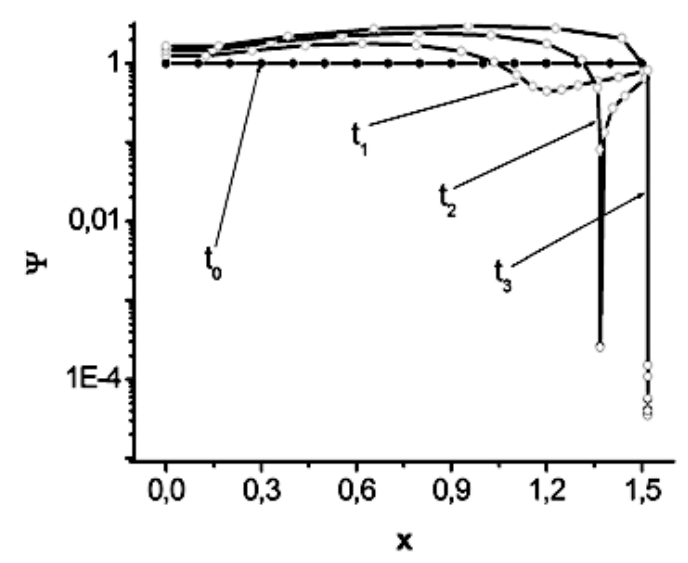

Fig. 3.4.

The strong nonlinearity of the equation in combination with a slow diffusion promotes the formation of large gradients inside the domain, which affects the redistribution of the grid points. In a short period of time, the space grid size near the nonlinear front decreases by three orders of magnitude while increasing by a factor of 25 in the region of the slow variation of the solution. The dynamics of the grid points is characterized by their trajectories (see Fig. 3.5). Nine of the $N=16$ points are concentrated on the front.

The coefficient $\alpha$ is a nonlinearity parameter; its value determines the location and time of the front formation. When $\alpha=0$, Eq. (10) is linear, the solution has no fronts, and the 
grid points remain fixed. When $\alpha$ increases, the region of the nonlinear front formation shifts to the left, and its amplitude increases. Accordingly, more grid points are concentrated near the front. The dynamics of the solution and of the grid points for $\alpha=2.5$ is illustrated in Figs. 3.6, 3.7.

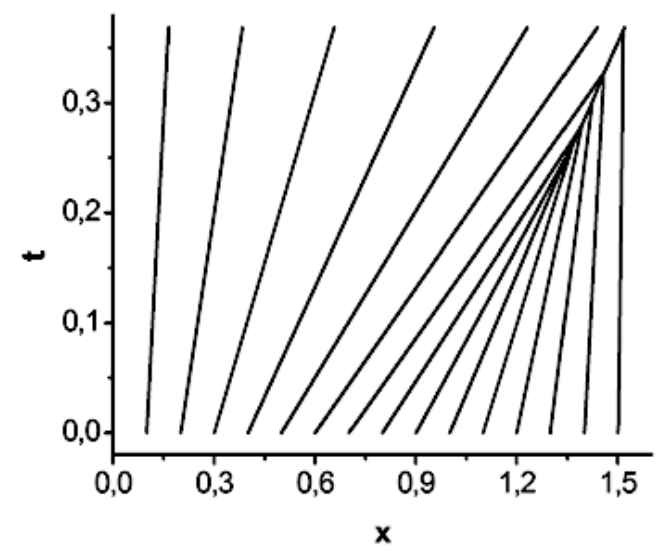

Fig. 3.5.

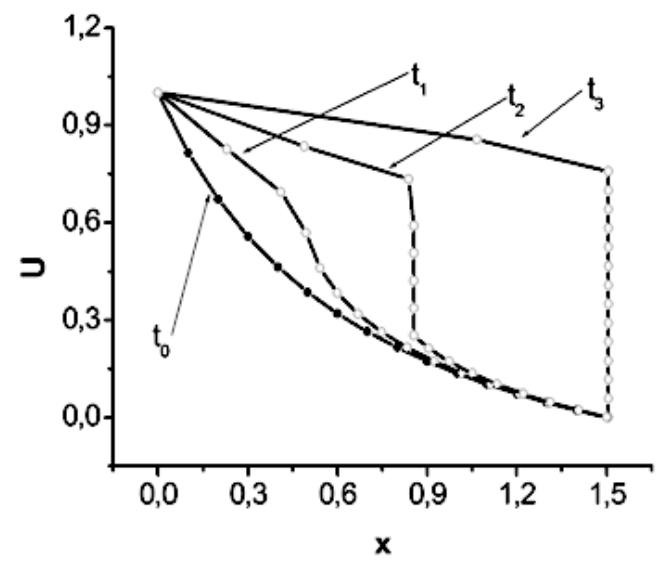

Fig. 3.6.

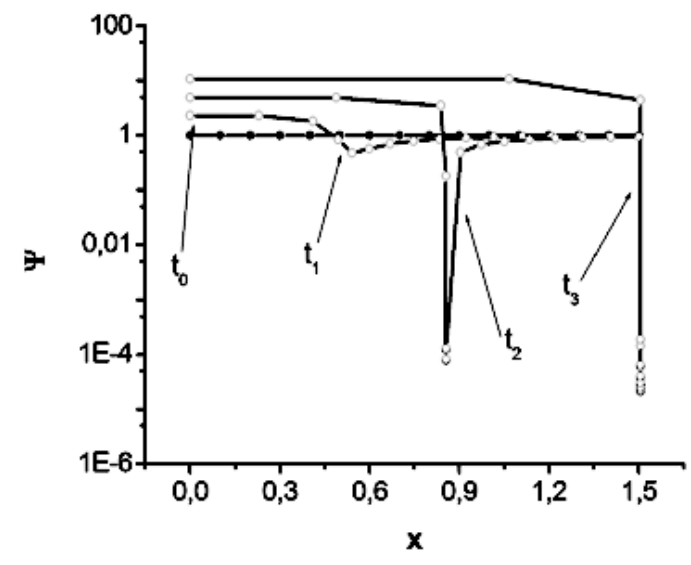

Fig. 3.7.

3.3.3. Burgers equation. The complete Burgers equation provides a model for the behavior of nonlinear waves, which typically contain one or several steep fronts. The difficulty of solving model Burgers problems numerically is well known (see [23]).

In terms of the variables $(x, t)$, the Burgers equation has the form

$$
\begin{gathered}
\frac{\partial u}{\partial t}+\frac{\partial\left(u^{2} / 2\right)}{\partial x}=\frac{\partial}{\partial x} \mu \frac{\partial u}{\partial x}, \quad \frac{\partial P(u)}{\partial u}=u, \\
x_{0}<x<x_{\infty}, \quad t>0
\end{gathered}
$$

Consider the typical statement of the problem in which the solution to the Burgers equation tends to a discontinuous solution because the physical viscosity $\mu=10^{-5}$ is small. Due to the special choice of the initial space profile $u^{0}(x)$, the quasi-discontinuous solution appears in the middle of the domain and on its boundary. 
The initial distribution $u(x, 0)$ was represented by two positive sinusoidal half-waves

$$
u(x, 0)=u^{0}(x)= \begin{cases}\left|\sin \left(2 \pi x / x_{R}\right)\right|, & 0<x<x_{R} \\ 0, & x>x_{R}\end{cases}
$$

The boundary conditions were chosen in the form

$$
u\left(x_{0, t}\right)=u\left(x_{R, t}\right)=0 .
$$

Representing the function $W(u)$ in the form

$$
W(u)=\frac{u^{2}}{2}-\mu \frac{\partial u}{\partial x}
$$

the Burgers equation in the variables $(q, \tau)$ is reduced to system $(21),(22)$, which allows us to use the family of difference schemes (24) complemented with the finite difference relations

$$
W_{i+1 / 2}=\frac{u_{i+1 / 2}^{2}}{2}-\frac{\mu}{\psi_{i+1 / 2}} \frac{u_{i+1}-u_{i}}{h}, \quad Q_{i}=-\left(u_{i}-\frac{\mu}{h}\left(\frac{1}{\psi_{i+1 / 2}}-\frac{1}{\psi_{i-1 / 2}}\right)\right) .
$$

3.3.4. Modeling results. Under the influence of the convective component and the small physical viscosity $\mu=10^{-5}$, the solution tends to a discontinuous solution as time increases.

First, problem (28)-(30) was solved on the grid with $N=2000$ fixed nodes. Due the special choice of the initial space distribution $u^{0}(x)$, two steep fronts appear inside the domain. When the second-order difference scheme is used, parasitic oscillations appear near both fronts, which cannot be gotten rid of even with $N=5000$ (see Fig. 3.8).

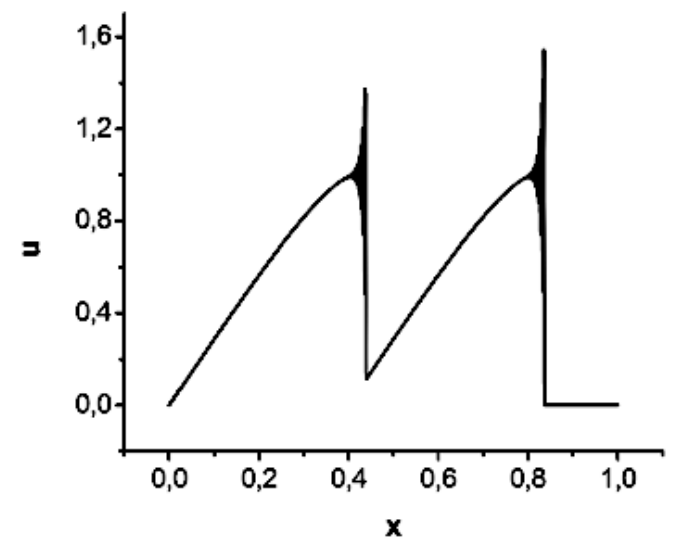

Fig. 3.8.

Figures 3.9-3.17 illustrate the solution of problem (21), (22), (29), (30) obtained at various instants of time using a dynamically adapted grid with $N=25$ grid points.

The calculations were performed using the second-order scheme (24),(31). Figures 3.9, 3.10 show the initial distribution of the functions $\psi\left(t_{0}, x\right), u\left(t_{0}, x\right)$. The calculations have shown that fronts of the solution begin to form on right edge of each half-wave. 


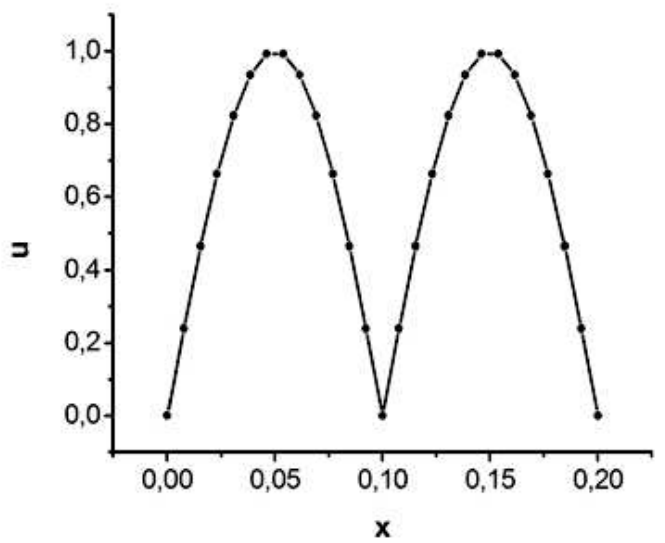

Fig. 3.9.

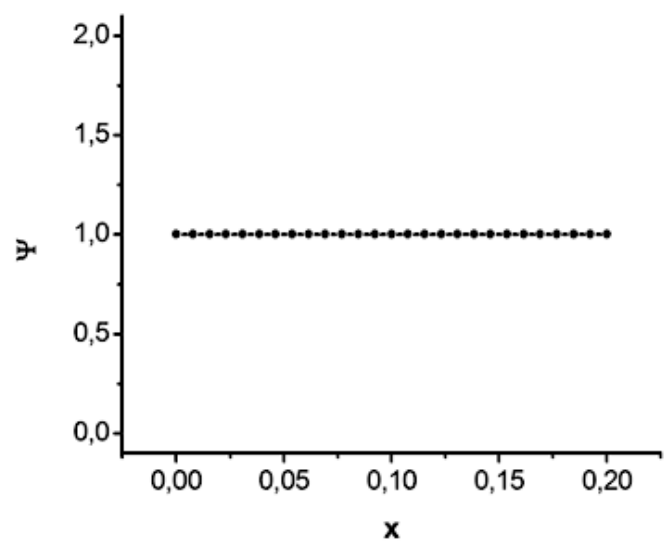

Fig. 3.10.

The grid responses to the appearance of large gradients by increasing the number of nodes in the neighborhood of the right boundaries of the half sinusoids $\left(\psi\left(t_{1}, x\right), u\left(t_{1}, x\right)\right.$ in Fig. 3.11, 3.12.

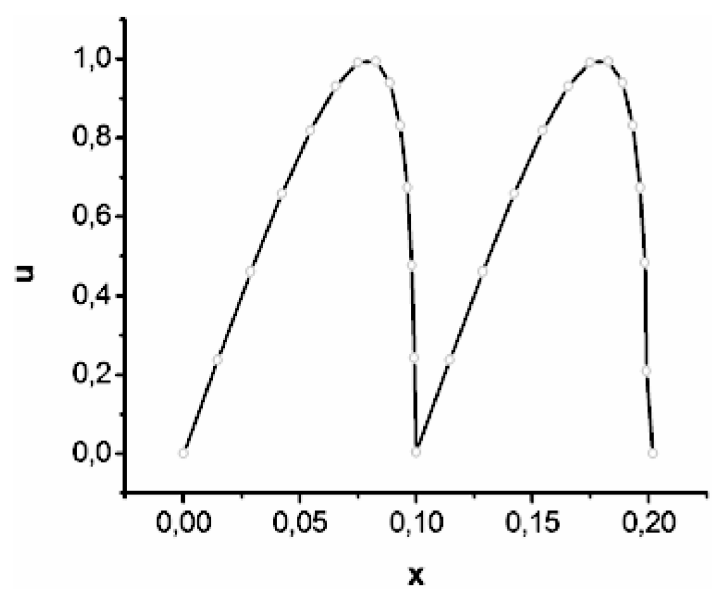

Fig. 3.11.

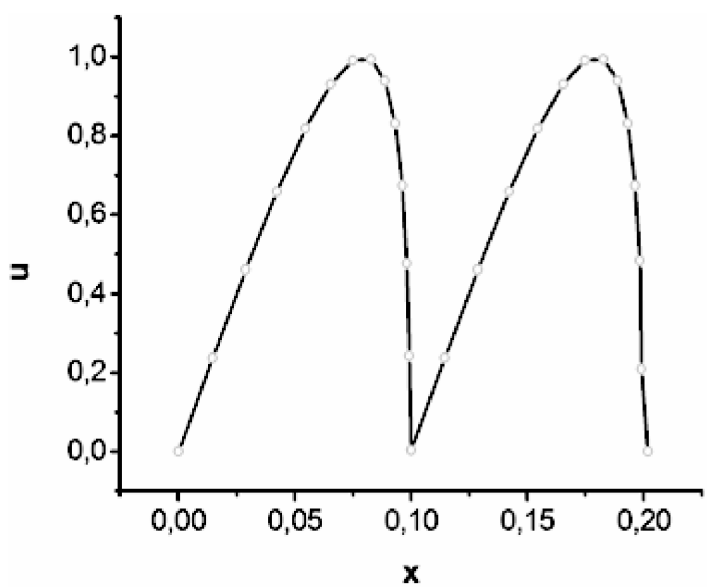

Fig. 3.12.

By the time when two fronts begin to form, the grid size decreases by several orders of magnitude. With time, the left front catches up with the right front (curves $\psi\left(t_{2}, x\right), u\left(t_{2}, x\right)$ in Fig. 3.13, 3.14), and then it absorbs the right front (curves $\psi\left(t_{3}, x\right), u\left(t_{3}, x\right)$ in Fig. 3.15, $3.16)$.

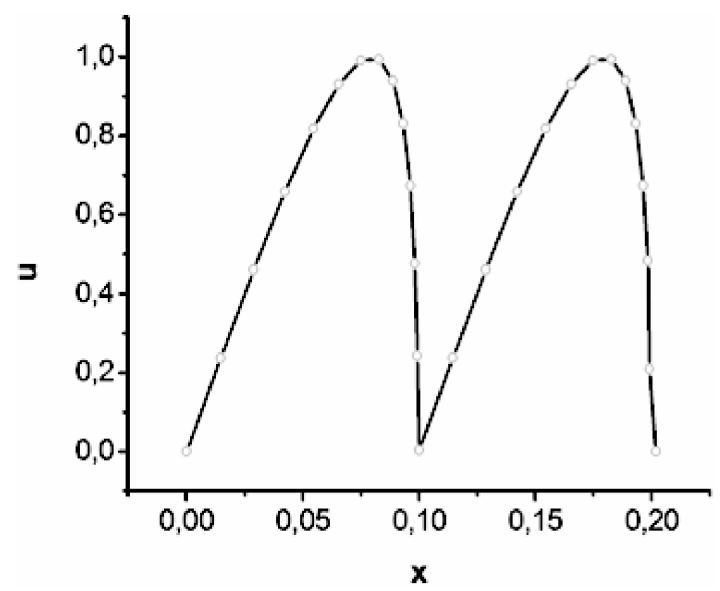

Fig. 3.13.

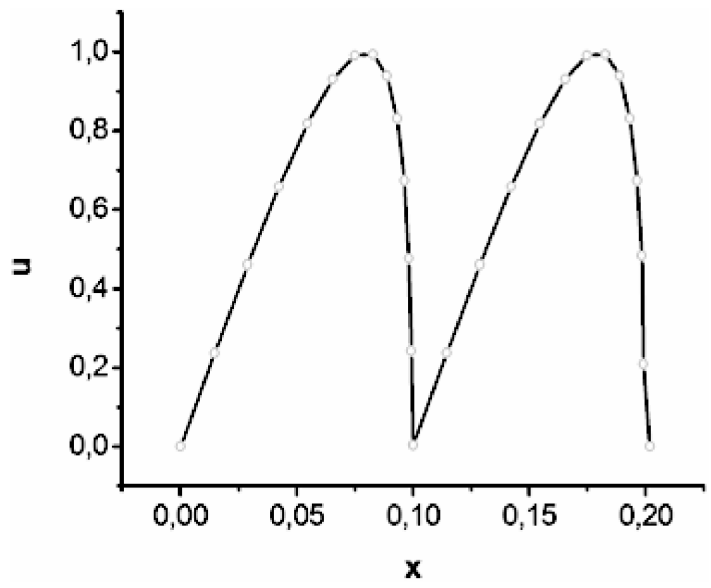

Fig. 3.14 . 


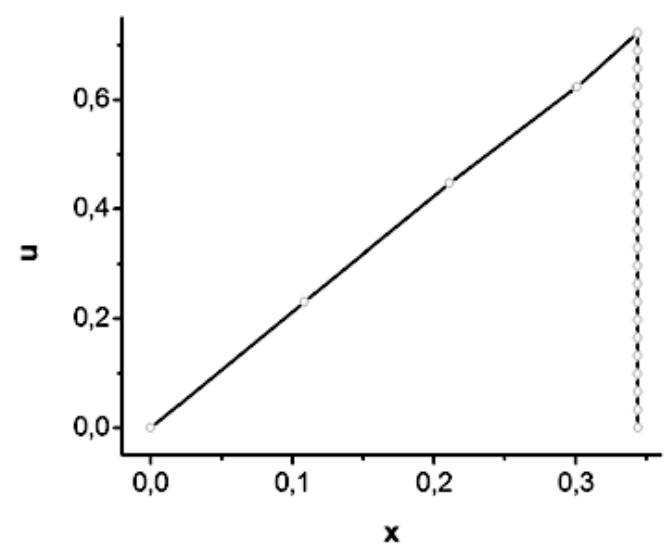

Fig. 3.15.

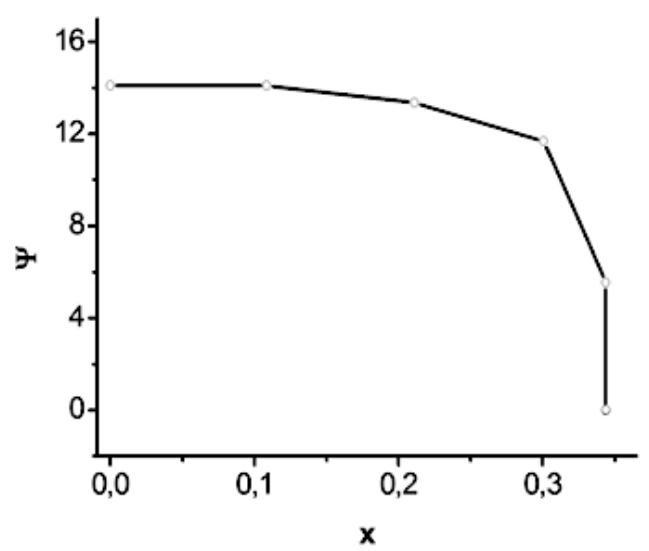

Fig. 3.16.

Later, the triangular shape remains along with the propagation in the mode with a single front. Most of the grid points concentrate on the front of the solution. The dynamics of the formation of fronts and their merge are best seen in the diagram of the node motion (see Fig. 3.17).

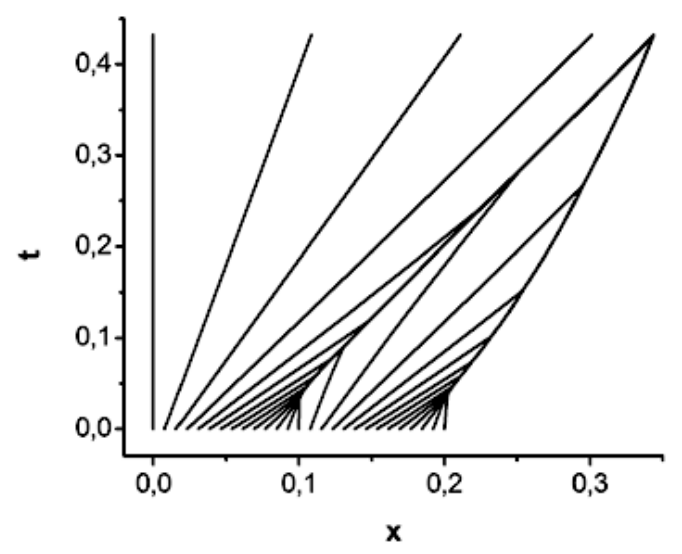

Fig. 3.17.

The complete absence of oscillations is achieved due the relocation of nodes fitted to the solution; as a result, the variation of the schemes completely vanishes. Due to this property of the difference schemes written in time-dependent systems of coordinates, dynamically adapted grids require an unusually small number of grid points.

In summary it is necessary to notice that the stated approach to the construction of dynamically adaptable grids is easily generalized to the case of multidimensional statements. In $[24,25]$, two model nonstationary problems of the phase front distribution into an interface in a two-dimensional statement have been considered. For solving one of them, grids with a uniform distribution along each of their directions were used, and for solving other problem grid with a strong condensation in the field of the large gradients were used. Applied problems of influence of the concentrated energy streams have been considered in [26, 27]. Two-dimensional dynamic grids have been constructed with the help of the diffusive approach. 


\section{Conclusions}

In this paper, we have performed a detailed analysis of the capabilities of the dynamic adaptation method as applied to the numerical solution of parabolic partial differential equations. Generalization of the results obtained for the nonlinear heat conduction and convection - diffusion equations shows that the idea of using the unknown solution for generating adapted grids makes the dynamic adaptation method universal, efficient, and algorithmically simple.

Universality is ensured by the use of an arbitrary time-dependent system of coordinates whose velocity depends on and is determined by the unknown solution. The efficiency is ensured by automatically fitting the velocity of the grid point motion to the dynamics of the solution.

This is achieved using the optimal transformation function. The components of the contracting and repulsive actions are contained in the original equation itself, and they are introduced in the control function using the quasi-stationarity principle. The close relationship of the adaptation mechanism with the structure of the parabolic equation makes it possible to perform automatic control of the grid points relocation preventing their trajectories from intersecting. This mechanism is inherent in all parabolic equations in contrast to the hyperbolic equations whose structure does not contain repulsive components.

The algorithmic simplicity is achieved by a universal approach to the generation of adapted grids independently of the type of the differential equation.

Acknowledgments. This work was supported by the Russian Foundation for Basic Research, project nos. DFG-08-07-91950 and 07-07-00045.

\section{References}

1. J.F. Thompson, Numerical Grid Generation, unknown place, North-Holland, 1982.

2. J.F.Thompson, Z. U.A. Warsi, and C.W. Mastin, Numerical Grid Generation. Foundation and application, New-York, North-Holland, 1985.

3. I. Babuska, J.E. Flaherty, W. D. Henshawand, and J. E. Hopcroft, Modeling, Mesh Generation and Adaptive Numerical Methods for Partial Differential Eqs., Springer, 1995.

4. H. C.Elman, Proceedings of the 4-9 International Conferences on Numerical Grid Generation in Computational Field Simulations, Ph.D. thesis, Department of Computer Science, Yale University, New Haven, CT, 1982.

5. Applied Geometry, Grid Generation and Highly Accurate Computations, Ed. by Yu. G. Evtushenko, M. K. Kerimov, and V. A. Garanzha. Vychisl. Tsentr Ross. Akad. Nauk, Vol. 1., Moscow, 2004.

6. N.A.Dar'in and V.I. Mazhukin, Approach to Generation of Adaptive Grids, Dokl. Akad. Nauk SSSR. 298 (1988), pp. 64-68.

7. N. A. Dar'in and V. I. Mazhukin, An Approach to the Generation of Adaptive Grids for Nonstationary Problems, Zh. Vychisl. Mat. Mat. Fiz. 28 (1988), pp. 454-460.

8. Yu. I. Shokin, First Differential Approximation, Nauka, Novosibirsk, 1979. [in Russian]

9. N. A. Dar'in, V.I. Mazhukin, and A.A.Samarskii, A Finite Difference Method for Solving OneDimensional Gas Dynamics Problems on Adaptive Grids, Dokl. Akad. Nauk SSSR. 302 (1988), pp. 10781081.

10. V. I. Mazhukin and L. Yu. Takoeva, Principles of Generation of Dynamic Grids That Adapt to Solutions of One-Dimensional Boundary Value Problems,Mat. Modelir. 2 (1990), no. 3, pp. 101-118.

11. V. I. Mazhukin, A. A. Samarskii, O. Kastel'yanos, and A. V.Shapranov, The Dynamic Adaptation Method for Nonstationary Problems with High Gradients, Mat. Modelir. 5 (1993), no. 4, pp. 32-56.

12. P. V. Breslavskii and V. I. Mazhukin, The Dynamic Adaptation Method in Gas Dynamics, Mat. Modelir. 7 (1995), no. 12, pp. 48-78. 
13. W. H. Hui, P.Y.Li, and Z.W.Li, A Unified Coordinate System for Solving the Two-Dimensional Euler Equations, J. Comput. Phys. 153 (1999), pp. 596-637.

14. W. H. Hui and S. Kudriakov, A Unified Coordinate System for Solving the Three-Dimensional Euler Equations, J. Comput. Phys. 172 (2001), pp. 235-260.

15. A. N. Gil'manov, Application of Dynamically Adaptive Grids to the Analysis of Flows with a Multiscale Structure, Zh. Vychisl. Mat. Mat. Fiz. 41 (2001), pp. 311-326.

16. D. V. Rudenko and S.V. Utyuzhnikov, Use of Dynamically Adaptive Grids for Modeling ThreeDimensional Unsteady Gas Flows with High Gradients,Zh. Vychisl. Mat. Mat. Fiz. 42 (2002), pp. 395-409.

17. H. Tang and T. Tang, Adaptive Mesh Methods for One- and Two-Dimensional Hyperbolic Conservation Laws, SIAM J. Numer. Anal. 41 (2003), pp. 487-515.

18. P. V. Breslavskii and V. I. Mazhukin, Dynamically Adapted Grids for Interacting Discontinuous Solutions, Zh. Vychisl. Mat. Mat. Fiz. 47 (2007),pp. 717-737.

19. M. M. Demin, V.I. Mazhukin, and A.A.Shapranov, Dynamic Adaptation Method for a Laminar Combustion Problem, Zh. Vychisl. Mat. Mat. Fiz. 41 (2001), pp. 648-661.

20. M. M. Demin, A.V.Shapranov, and I.Smurov, The method of construction dynamically adapting grids for problems of unstable laminar combustion, Numerical Heat Transfer, Part B: Fundamentals. 44 (2003), no. 4, pp. 387-415.

21. D. A. Anderson, J. C. Tannehill, and R. H. Pletcher, Computational Fluid Mechanics and Heat Transfer, New York.: Hemisphere Publishing Corporation, 1984.

22. J. B. Bell and G. R. Shubin, An adaptive grid finite difference method for conservation law, J. Comput. Phys. 52 (1983), pp. 569-591.

23. E. R. Benton and G.W.Platzman, A table of the one-dimensional Burgers equation, Quarterly of Applied Mathematics. 30 (1972), pp. 195-212.

24. N. A. Dar'in, V. I. Mazhukin, and A. A. Samarskii, Finite-difference method for solving nonstationary two-dimensional boundary problems on adaptive grids dynamically related with solution, Preprint of Keldysh Institute of Applied Mathematics of USSR Academy of Sciences, no. 117, 1987, p. 27.

25. N. A. Dar'in and V. I. Mazhukin, Mathematical modeling of nonstationary two-dimensional boundary problems on grids with dynamic adaptation, Mathematical Modeling. 1 (1989), no. 3, pp. 29-43.

26. V. I. Mazhukin, A. A. Samarskii, and M. M. Chuiko Dynamic adaptation method for numerical solving nonstationary multi-dimensional Stefan problems, Dokl. Ross. Akad. Nauk. 368 (1999), no. 3, pp. 307310 .

27. V.I. Mazhukin and M. M. Chuiko Solution of $l$ multi-interface Stefan problem by the method of dynamic adaptation, Computation Methods in Applied Mathematics. 2 (2002), no. 3, pp. 283-294. 